\title{
Mixed Matrix Membrane Performance Prediction for Gas Separation using Modified Models
}

\author{
A. Salimi ${ }^{1}$, O. Bakhtiari ${ }^{2}$, M. K. Moghaddam ${ }^{3}$ \& T. Mohammadi ${ }^{4 *}$ \\ ${ }^{1 \& 4}$ Department of Chemical Engineering, South Tehran Branch, Islamic Azad University, P. O. Box 11365-4435, \\ Tehran, Iran \\ ${ }^{2 \& 3}$ Department of Chemical Engineering, Razi University, Kermanshah, Iran Department of Chemical \\ Engineering, Iran University of Science and Technology, Tehran
}

\begin{abstract}
Gas separation using membrane processes are potentially economical in industrial scale. Two parameters are used for analyzing the membrane separation performance: permeability and selectivity. There is a trade off between them for polymeric membranes that makes it impossible to increase both of them simultaneously. Molecular sieve membranes, on the other hand, exhibit high permeability and selectivity but are brittle in nature and costly. A new generation of membranes has made many hopes to use simultaneously both desired properties of polymers and molecular sieves in a structure called "mixed matrix membrane (MMM)" where a molecular sieve is incorporated within a polymer matrix. As other branches of science and engineering, having a tool to predict MMMs performance seems to be essential to save time and money for research and industrial applications. Many mathematical models were developed to predict MMMs performance based on separation performance of fillers and polymers. Maxwell model is the simplest model developed for prediction of electrical properties of composite materials but it is not perfect for all cases. Some modifications were performed on Maxwell model and some other modified models were developed for better prediction of MMMs separation performance. In this research, modified Maxwell and Bruggeman models were employed to predict gas separation performance of some MMMs in the current work and the results were acceptable for all non-ideal cases which might be occurred in MMMs structure.
\end{abstract}

Keywords: Mixed matrix composite membrane, gas separation, ideal and modified models

\subsection{INTRODUCTION}

Gas separation using membranes is one of the very fast growing branches in separation science. In membrane-based gas separation processes, mixtures are separated to their components by differential permeation through the membranes. A number of advantages, including low capital and operating costs, low energy requirements and generally ease of operation are offered by membrane separation processes [1-3].

\footnotetext{
* Corresponding to: T. Mohammadi (email: torajmohammadi@iust.ac.ir)
}

Current applications of membrane-based gas separation include oxygen and nitrogen enrichment, hydrogen recovery and natural gas sweetening: In the area of membrane-based gas separation, non-porous polymeric membranes based on solution-diffusion mechanism have been exclusively employed in current commercial devices $[2,3]$.

Membranes economics is closely linked to their transport properties: permeability, which determines separation productivity; and selectivity, which determines separation 
efficiency. According to the solution-diffusion model, permeation of molecules through the membranes is controlled by two major parameters: diffusivity coefficient $(D)$ and solubility coefficient $(S)[1,2]$;

$$
P=D \times S
$$

Permeability $(P)$ represents the ability of molecules to pass through a membrane. The ability of a membrane to separate two different molecules of $A$ and $B$, is called "membrane selectivity" is defined as $[1,2]$ :

$$
\alpha_{A B}=P_{A} / P_{B}
$$

Where $\alpha_{A B}$ is the membrane selelectivity and $P_{A}$ and $P_{B}$ are permeability of molecules $A$ and $B$. Rewriting 1 and 2 results in:

$$
\alpha_{A B}=\left(D_{A} / D_{B}\right) \times\left(S_{A} / S_{B}\right)
$$

For polymeric membranes, there is a tradeoff between permeability and selectivity, which does not let to increase both permeability and selectivity simultaneously, as reported for the first time by Robeson in 1991 as upper bound limit [4]. This limit of polymeric membranes for the $\mathrm{O}_{2} / \mathrm{N}_{2}$ separation is shown in Figure $1[1,2]$.

As depicted in Figure 1, inorganic membranes have separation performances well above the Robeson upper bound limit. However, they are brittle in nature and expensive to make industrial scale defect-free membrane surface areas [2].

A recent attended old approach is to incorporate inexpensive fine molecular sieves particles into polymer matrices to employ both desired properties of filers and polymers to pass the Robeson upper bound limit. As mentioned above, polymeric membranes are limited in tailor made reconstruction of their structure. Restriction in their chain mobility in a tough structure via i.e. cross linking results in better diffusivity selectivity ( $D$ in 1 ), while solubility selectivity ( $S$ in 1 ) decreases and vice versa. Incorporation of fillers within polymer matrices results better diffusivity selectivity, while solubility selectivity remains nearly constant and the separation mechanism moves from the solution-diffusion to the molecular sieving.

MMMs offer a new type of membranes which are able to provide a step-change in membrane performance to exploit existence of separation potentials economically specially in gas separation field and too many researches are focused on this type of membranes. Having a powerful tool to predict MMMs separation performance can save time and money and accelerate the research trend to achieve attractive economical areas (Figure 1).

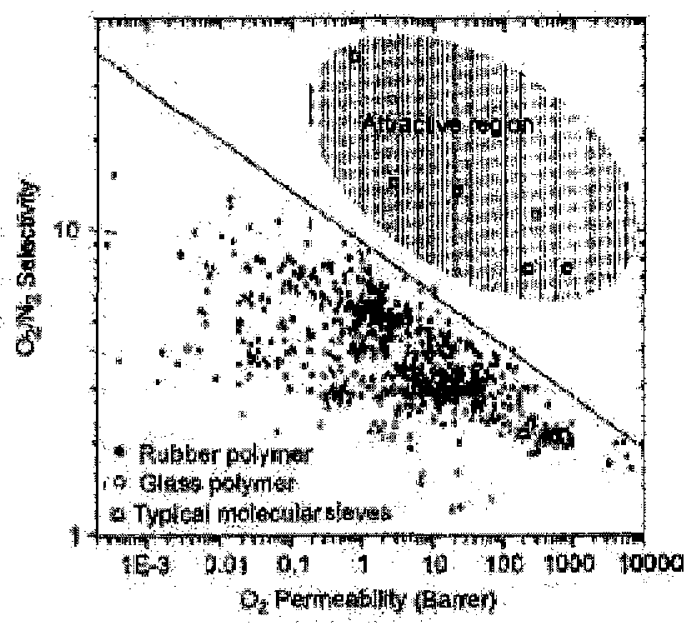

Figure 1 Selectivity and permeablity tradeoff for polymeric membranes and separation performance of inorganic membranes for $\mathrm{O}_{2} / \mathrm{N}_{2}$ separation [2]

\subsection{DEVELOPED MODELS FOR MMMS PERFORMANCE PREDICTION}

For optimized use of the MMMs in gas separation processes, having knowledge of the relation between MMMs separation performance and their filler type and content is necessary to save time and money. The simplest and earliest model was based on adaptations of thermal/electrical conductivity models. Since there is a close analogy between thermal/electrical conduction in composite materials and permeation of species through such materials, conductivity models have been readily adapted to permeability of species in MMMs [2]. 


\subsection{Maxwell Model}

Maxwell model, originally developed in 1873 to predict electrical conductivity of particulate composites, was adapted to permeability as follows $[1,5]$ :

$$
P_{M M M}=P_{c}\left[\frac{P_{d}+2 P_{c}-2 \phi_{d}\left(P_{c}-P_{d}\right)}{P_{d}+2 P_{c}-\phi_{d}\left(P_{c}-P_{d}\right)}\right]
$$

Where $P_{M M M}$ is the effective permeability of MMM, $P$ is the permeability, $\phi$ is volume fraction and $c$ and $d$ present continuous and dispersed polymeric phases, respectively [1].

As observed, this ideal model has three parameters: permeabilities of dispersed and continuous phases and filler loading and doesn't consider non-ideal cases (defects) which may occur in the MMMs structure and can be applied for limited cases of $\phi<0.2$ [6]. For higher filler loading, significant deviations are expected between model predictions and experimental data [6].

Proper polymer and filler selection, pretreatment, preparation and post-treatment procedures are of the most important key features in successful ideal MMMs preparation $[7,8]$. Filler size and content and polymer concentration are also other affecting parameters on filler distribution inside polymer matrix and MMMs separation performance [7-9]. Void formation around incorporated fillers, fillers pores blockage by polymers and polymer chains rigidification are undesired phenomena that can potentially occur in MMMs preparation especially for glassy polymers and result in non-ideal MMMs morphology $[2,7,10,11]$. Different morphologies may be encountered in MMMs as shown schematically in Figure 2 [2]. Thus, the performance predictive models should be able to consider these non-ideal cases, which are neglected by Maxwell model.

\subsection{Bruggeman Model}

Bruggeman model was originally developed as a differential effective medium approach for dielectric constant of particulate composites and
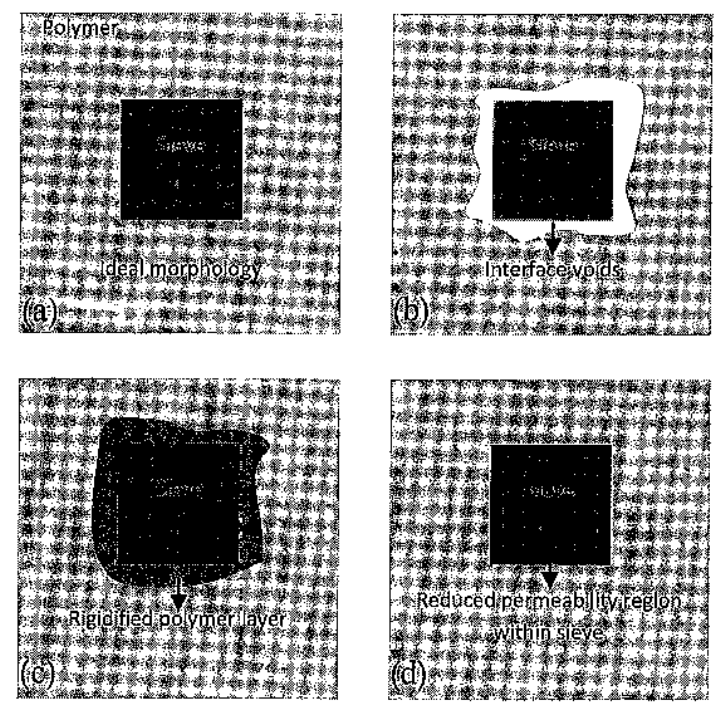

Figure 2 Ideal and different non-ideal cases which may be encountered in MMMs preparation [2]

could be adapted to permeability as well as follows $[6,12]$ :

$$
\left(P_{r}\right)^{1 / 3}\left[\frac{\lambda_{D C}-1}{\lambda_{D C}-P_{r}}\right]=(1-\phi)^{-1}
$$

Where $P_{r}$ is reduced MMM permeability by polymer permeability and $\lambda_{D C}$ is the permeability ratio of dispersed phase over that of continuous phase.

Although, Bruggeman model is more accurate than Maxwell model [6], it has some similar limitations. In addition, this model is not explicit for permeability. Predictions of Maxwell and Bruggeman models for zeolite 4A and PVAc MMMs are shown in Figure 5. As observed, the results are so far from the experimental data and this indicates the need for more accurate models [13]. Employing the modified Maxwell model for the same separation system as shown in the following sections, reveals better fitting with the experimental data. 


\subsection{Pal Model}

Pal model was developed based on the Bruggeman model taking into account packing efficiency of filler particles incorporation. This model considers a factor of $\phi_{m}$ for maximum volume fraction of filler distributed in random manner $[5,6]$ and all other filler volume fractions are compared with $\phi_{m}$. This enables the model to predict MMMs permeation correctly especially at $\theta \rightarrow \theta_{m}$. It should be noted that this model reduces to Bruggeman model when $\theta_{m}=1$.

$$
\left(P_{r}\right)^{1 / 3}\left[\frac{\lambda_{d c}-1}{\lambda_{d c}-P_{r}}\right]=\left(1-\frac{\phi}{\phi_{m}}\right)^{-\phi m}
$$

Where $\phi_{m}=0.64$ demonstrates random close packing of uniform spheres within the polymer matrix.

Bruggeman and $\mathrm{Pal}$ models are implicit models and should be solved using numerical methods. In current study, the fixed point method was used to solve the model equations. Depending on the permeabilities magnitude case study, one of the following equations was used to solve the models depend on the magnitudes of $P_{r} \phi$ and $\lambda_{D C}[14]$ :

$$
\begin{aligned}
& P_{r}=\left[(1-\phi)^{-1} /\left(\frac{\lambda_{d c}-1}{\lambda_{d c}-P_{r}}\right)\right]^{3} \\
& P_{r}=\lambda_{d c}-\left(P_{r}\right)^{1 / 3} \times\left(\lambda_{d c}-1\right) /(1-\phi)^{-1}
\end{aligned}
$$

Where the error of the $P_{r}$ should be less than $10^{-3}$.

\subsection{ACCURACY OF THE IDEA MODELS PREDICTIONS}

The results of the Ultem - CMS MMM, reported by De. Q. Vu et al. [15] were used to compare the accuracy of these ideal models. As can be seen in Figure 3 the experimental results of which type of the MMM exhibit an ideal trend line. This indicates that there is no non-ideal morphologies and the results of Maxwell model are close enough to the experimental results and followed by Bruggeman and Pal models which predict the experimental results with less accuracy. To compare the modified models prediction accuracy, percentage of average absolute relative error (AARE\%) was defined as:

$$
A A R E \%=\frac{100}{N} \sum_{i=1}^{N} \mid \frac{P_{i}^{c a l}-P_{i}^{\exp }}{P_{i}^{\exp }}
$$

Where $\mathrm{N}$ is the number of experimental data and $P_{i}^{e x p}$ is the experimental permeability of the component $i$ and $P_{i}^{c a l}$ is the calculated permeability of component $i$.

The results of the AARE \% values for different cases are shown in Table 1 . Since the Pal and Bruggeman models are close together and there is no considerable improvement in the prediction ability of $\mathrm{Pal}$ model over the Bruggeman, the later was employed hereafter.

Table 1 AARE\% value of the ideal models in the current studies

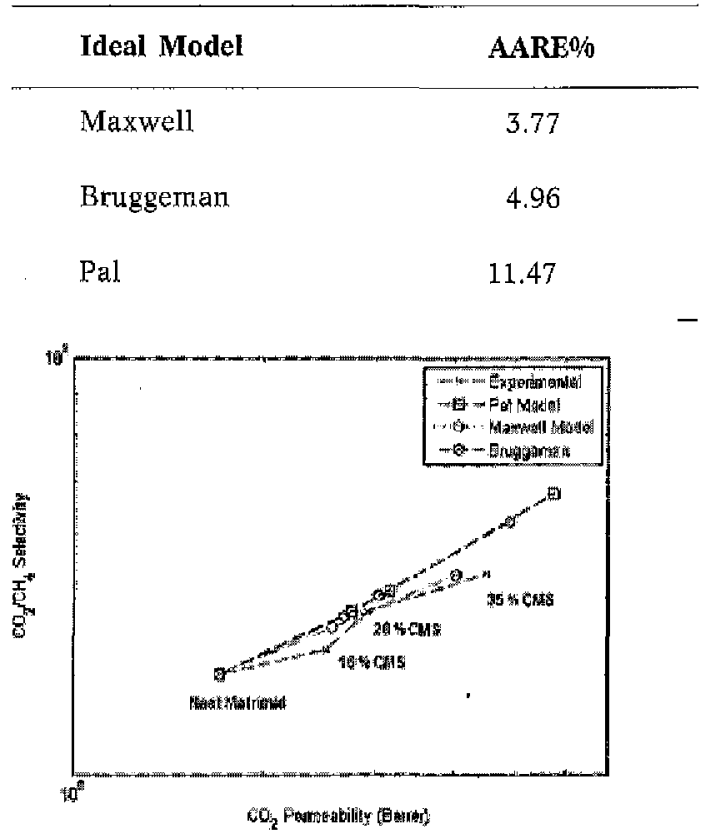

Figure 3 Comparison of the ideal models with the experimental data for the UltemCMS MMS for $\mathrm{CO}_{2} / \mathrm{CH}_{4}$ separation at 35 
As observed, the ideal Maxwell model prediction is in good agreement with the experimental and has less deviation compared with the other models of Bruggeman and Pal. However, the ideal models have relatively large deviation especially for non ideal cases usually encountered in prepared. MMMs [1] and some modification are required.

\subsection{MODIFICATION OF THE IDEAL MODELS}

As mentioned above, ideal models assume that there are just two phases in a MMM structure: a polymeric continuous phase and a dispersed filler particles phase and doesn't take into account for the above mentioned defects. In the modified models, a third phase of interface between polymer matrix and filler particles is considered and all three phases are taken into account for MMMs performance prediction. The third phase permeation and volume fraction should also be estimated to predict MMMs separation performance.

As mentioned above and observed in Figure 2 different interfaces of gap generation (Figure 2b), chain rigidification (Figure $2 \cdot c$ ) and pore blockage (Figure 2-d) can be distinguished depending on polymer and filler nature and possible modifications, MMMs preparation conditions and their pre- and/or post-treatment [2]. Separation performance of MMMs is also different from that of neat polymeric membranes regarding to the nature interface phase. In the ideal case, MMMs separation performance proportionally enhances with increasing filler loading (Figure 4, case 0) [1]. In the case of polymer chain rigidification around the filler particles due to immobilization of attached end of polymer chains to the filler surface, MMMs selectivity increases, while their permeability decreases comparing with the bullk polymer matrix (Figure 4, case I) [1]. In the case of void formation around the filler particles (the third plase in MMMs is gaps around the filler particles), depending on the relative size of voids and penetrants, permeability increases, while selectivity either remains constant (Figure 4, case II) or decreases based on Knudsen mechanism of molecular sieving (Figure 4, case III). The latter (Case III) is a special case of the former (case II), where the effective voids thickness is the same order as the size of penetrants $(\sim 5 A)$ [1]. In addition to these three cases, polymeric chains may be entered into the filler pores and block them totally (Figure 4, case IV) or partially (Figure 4 , case V). Cases IV and V are basically like case I but the interface phase is placed within the filler particles. In case IV, the strongly attached polymer

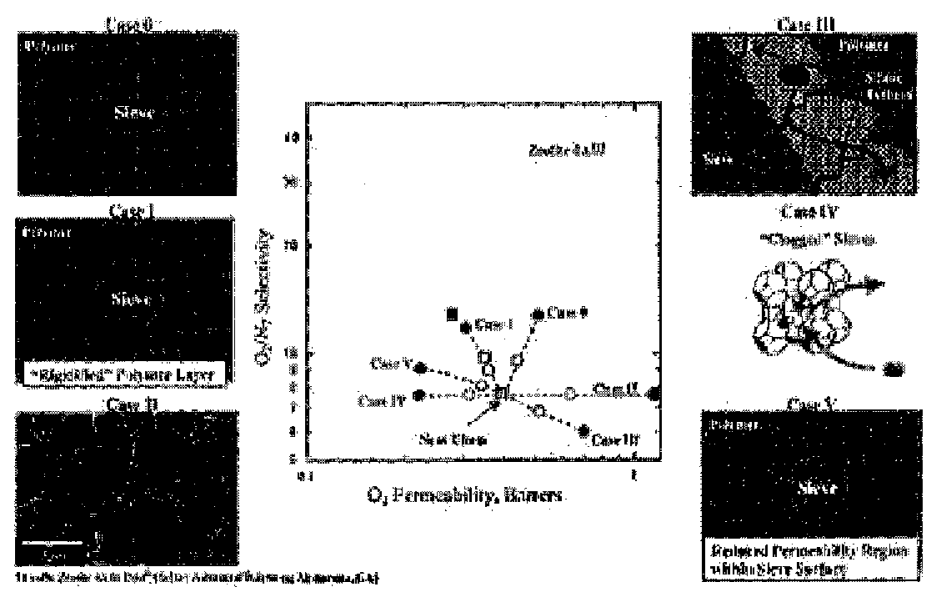

Figure 4 Zeolite 4A-Ultem MMMs transport properties vs. their morphologies. [O and $\square$ ] are representations of calculated and experimental values. [• and $\square$ are representations of 15 and 35 vol. $\%$ respectively [1] 
molecules, which are entered into the filler pores, completely prevent the penetrants of interest from entering the fillerpores, completely prevent the penetrants of interest from entering the filler pores, whereas in case $\mathrm{V}$, the penetrants of interest enter or pass through the filler slower than usual [1]. Obviously, both of these cases can result in abortive filler incorporation. The trend of ideal case has negative slope when the filler permeability is less than that of the polymer phase [16].

Considering these four non-ideal cases, ideal models should be modified in a manner to consider existence of the third phase in the four above mentioned cases. In one approach, ideal models can be used twice. At first, this model is applied for the filler phase as the dispersed phase and the interface phase as the continuous phase to obtain an effective permeability for the hypothetical new filler, $P_{e f f}$, which is formed from the filler phase surrounded by the interface layer (rigidified or void):

$$
P_{e f f}=P_{I}\left[\frac{P_{d}+2 P_{I}-2 \phi_{s}\left(P_{I}-P_{d}\right)}{P_{d}+2 P_{I}+2 \phi_{s}\left(P_{I}-P_{d}\right)}\right]
$$

And for Pal model the first application of the model leads to:

$$
\left(P_{\text {reff }}\right)^{1 / 3}\left(\frac{\lambda_{d I}-1}{\lambda_{d I}-P_{r e f f I}}\right)=\left(1-\phi_{s}\right)^{-1}
$$

Where $P_{I}$ is permeability of the interface phase and $\phi_{s}$ is volume fraction of the filler in the pseudo-disperse phase [1] and $P_{\text {reffI }}$ is the ratio of permeability of the pseudo disperse phase over permeability of interface phase and $\phi_{s}$ is volume fraction of filler in the pseudo-disperse phase and $\lambda_{d l}$ is the ratio of the permeability of a penetrate in disperse phase over its permeability in interface phase.

In another approach, the new hypothetical filler with its effective permeability and modified volume fraction is supposed to be incorporated into the polymer matrix.

\subsection{Modification of the Models for Polymer Chain Rigidification Around Filters (Case 1)}

It can be supposed that the rigidified chains prevent the penetrates to diffuse in filler particles and lead them to pass through lower resistance pathway of polymer matrix. This undesired case is equal to blending of rigidified interface polymer chains with the original polymer mattix to exhibit a middle separation performance or to crosslinking of polymer chains for higher selectivity and lower permeability. Robeson upper bound limit in interface layer aborts effectiveness of filler incorporation. In the case of polymer chain rigidification around the filler particles, two parameters are considered to describe the defect: the first one is a factor to show the chain rigidification reductive effect on permeability of the rigidified region and defined as chain immobilization factor, $\beta$. Then, permeability of the rigidified region compared with that of the polymer matrix is assumed to be reduced by this factor [1]:

$$
P_{I}=\frac{P_{c}}{\beta}
$$

Where $P_{I}$ is the interface permeability of the rigidified polymer chains [1]. The second parameter in this case is the thickness of rigidified region around the filler particles. The thicker rigidified region results in the lower diffusion rates of penetrates toward the more aborted filler particles [13].

In the first application of models for this case, the permeability of the penetrates through the pseudo-dispersed phase (the original fillers surrounded by the rigidified polymer layer) can be defined based on Equation 12.In this case, $\phi_{s}$ is defined as [13]:

$$
\phi_{s}=\frac{\phi_{d}}{\phi_{d}+\phi_{i}}
$$

By assuming spherical fillers, $\phi_{s}$ can be written as: 


$$
\phi_{s}=\frac{r_{d}^{3}}{\left(r_{d}+l_{i}\right)^{3}}
$$

Where $r_{d}$ and $l_{i}$ are spherical fillers radius and rigidified layer thickness, respectively. Now, MMM's permeability $\left(P_{3 M M}\right)$ is calculated by the second application of Maxwell model considering pseudo filler particles in the polymer matrix [1]:

$$
P_{3 M M}=P_{c}\left[\frac{P_{e f f}+2 P_{c}-2\left(\phi_{d}+\phi_{i}\right)\left(P_{c}-P_{e f f}\right)}{P_{e f f}+2 P_{c}+\left(\phi_{d}+\phi_{i}\right)\left(P_{c}-P_{e f f}\right)}\right]
$$

And for Pal model the second application of the model leads to:

$$
\left(P_{r 3 m m c}\right)^{1 / 3}\left(\frac{\lambda_{e f f c}-1}{\lambda_{e f f c}-P_{r 3 m m c}}\right)=\left(1-\left(\phi_{d}+\phi_{I}\right)\right)^{-1}
$$

Where $\phi_{i}$ is volume fraction of the interface in the whole of MMM bulk [1] and $P_{r 3 m m c}$ is the ratio of permeability in the whole of MMM over permeability of the continuous phase and $\lambda_{\text {effc }}$ is the ratio of permeability in the pseudo disperse phase over its permeability in the neat polymer phase

$$
\phi_{i}=\left(\frac{\left(r_{d}+l_{i}\right)^{3}-r_{d}^{3}}{r_{d}^{3}}\right) \times \phi_{d}
$$

\subsection{Modification of the Models for Void Formation around Fillers (Cases II and III)}

In the case of voids formation around the filler particles (cases II and III), two parameters are needed to predict MMM's separation performance, permeability of the penetrants through the voids interface and thickness of the voids around the filler particles. Permeability of the voids around the filler particles is assumed to be the product of Knudsen diffusion coefficient through a pore with the same hydraulic diameter as the void thickness and sorption coefficient of the penetrants in the void. The sorption coefficient in the voids is assumed to be the same as that in the gas phase [17]

$$
\begin{aligned}
& D=D_{k \text { kndsen }}\left(1-\frac{\sigma_{p}}{2 l_{i}}\right) \\
& D_{\text {knudsen }}=\left(\frac{d_{\text {pore }}}{3}\right)\left(\frac{8 R T}{\pi M_{i}}\right)^{1 / 2} \\
& S=\frac{1}{R T}\left(1-\frac{\sigma_{p}}{2 l_{i}}\right)
\end{aligned}
$$

Where $D$ and $D_{k n u d s e n}$ are diffusion coefficient in the voids and Knudsen diffusion coefficient of penetrants, respectively, $\sigma_{p}$ is the kinetic diameter of penetrants, $l_{i}$ is the void thickness around the fillers, $d_{\text {pore }}$ is the equivalent pore of the void thickness, $R$ is the universal gas constant, $T$ is absolute temperature, and $M_{i}$ is the penetrants molecular weights. For calculation of Knudsen diffusion coefficient through the interface voids it is assumed that [1]:

$$
d_{\text {pore }}=2 l_{i}
$$

The terms in the parentheses in Equations 18 and 20 must be added to account for the finite size of penetrants when the voids dimension is the same order of magnitude as the penetrants diameter (case III) [17]. Now using Equation 1, permeability of the interface can be written as:

$$
P_{I}=D \times S
$$

The thickness of interface voids, $l_{i}$ is also the second parameter in this case at constant pressure and temperature, and $l_{i}$ is the main affective parameter in the case of voids formation around the filler particles [17]. As observed in Figure 4, penetrants in this case can freely move around the filler particles by convective flow, and this will results in increasing permeability, while selectivity may be potentially reduced dramatically. The interface voids is formed due to weak wettability of the fillers surface, residual stresses duration MMMs preparation, etc especially for glassy polymers $[2,7,10,11,18]$. 


\subsection{Modification of the Models for Polymer Chain Entered into Around Fillers (Cases IV and V)}

Similar to those of case I, there are two parameters in these cases. Moore et al. introduced the first parameter of permeability reduction factor for this case $\beta$, the same as chain rigidification factor in case $I$, where the polymer matrix permeabilities of penetrants are divided by. The second parameter in this case is the thickness of blocked region inside the filler particles [13]. Permeability of the interface is given by:

$$
P_{I}=\frac{P_{c}}{\beta}
$$

Volume of the rigidified polymer fraction within the filler particles is given by:

$$
\phi_{S}=\frac{\left(r_{d}-l_{i}\right)^{3}}{r_{d}^{3}}
$$

Where $l_{i}$ is the thickness of the interface phase, and $\phi_{s}$ is the volume fraction of the filler particles in the pseudo-disperse phase [13]. Permeability equation is the same as the equation in case I[1].

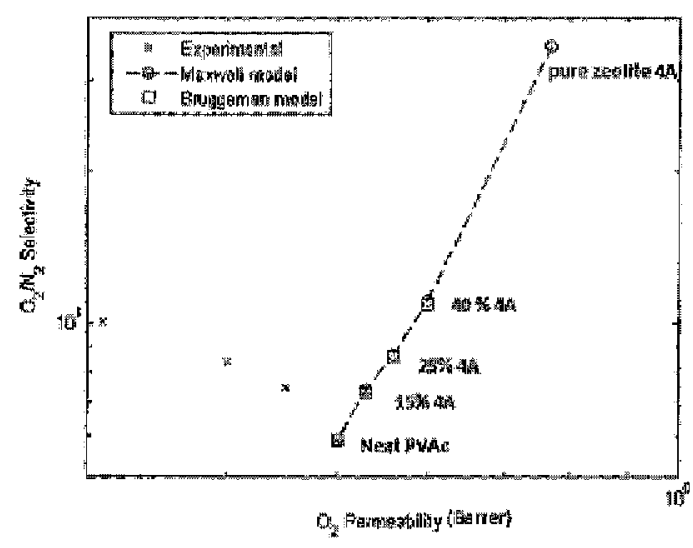

Figure 5 Comparison of Maxwell and Bruggeman models predictions with experimental data for $\mathrm{a}_{z} / \mathrm{w}_{z}$ separation [13]

\subsection{APPLICATION OF THE MODIFIED MAXWELL MODEL FOR PREDICTING SEPARATION PERFORMANCE OF THE MMMS}

\subsection{MMMs with Rigidified Interface}

\subsubsection{Prediction of MMMs with zeolite 4A Fillers Performance for $\mathrm{O}_{2} / \mathrm{N}_{2}$ Separation}

As observed in Figure 5, ideal models cannot predict the real cases encountered in MMMs structures. The modified Maxwell model was then employed to predict the experimental data of $\mathrm{O}_{2} /$ $\mathrm{N}_{2}$ separation. Literature experimental data was compared with Figure 4 to find the phenomena of void formation, chain rigidification, or pore blockage and then their numerical values were gathered using Get Data Graph Digitizer 2.22 software in the case of graph representation or read from reported tables [12]. After that by using the least square method, different cases parameters were found in such manner to minimize sum of gas pair permeabilities deviations from the experimental data. Programming was nerformed using MATLAB 2010a software.

Considering the MMMs separation erformance trend vs. filler loading regarding to 1ose shown in Figure 4, the non-ideal case can be scognized and proper modified Maxwell model or the case (e.g. void formation around the fillers, hain rigidification or polymer entrance into the llers pores) can be selected. Based on the least quare method, the best fitting parameters of the elected model can be determined and MMMs eparation performance can be predicted.

As reported by Moore et al. [13], by increasing eolite 4A loading in PVAc, Ultem and BAPBPADA polymer matrices, permeability of $\mathrm{O}_{2}$ aecreases, while $\mathrm{O}_{2} / \mathrm{N}_{2}$ selectivity increases continuously, as observed in Figure 6. Comparing these trends with those of different cases in Figure 4 , indicates that the polymer chain rigidification occurs and two parameters are needed to fit the modified Maxwell model to experimental data: chain rigidification factor, $\beta$, and thickness of reduced mobility region $\left(l_{i}\right)[1]$. Experimental data for each MMM was gathered and using the 


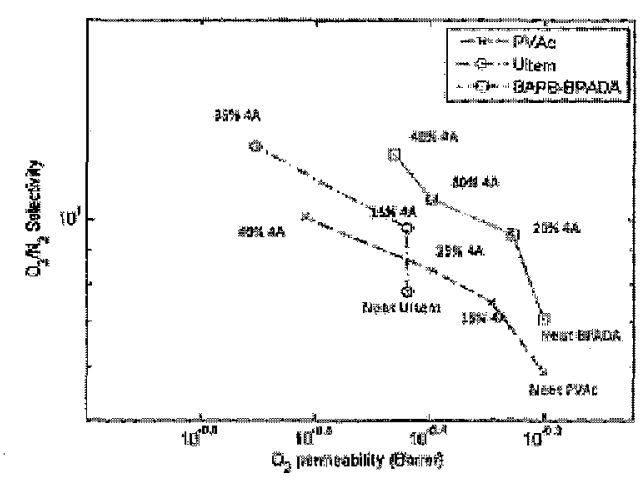

Figure 6 Experimental data for $\mathrm{O}_{2} / \mathrm{N}_{2}$ separation using zeolite $4 \mathrm{~A}$ filler in different. polymer matrices at $35^{\circ} \mathrm{C}$ [17]

least square method, the best fitted parameters with the experimental data were calculated in order to minimize the $\mathrm{O}_{2}$ permeabilities and $\mathrm{O}_{2} /$ $\mathrm{N}_{2}$ selectivities simultaneously. The results are reported in Figure 7. As observed, the modified Maxwell model predicts the experimental data with acceptable regression coefficients. Smaller regression coefficient for zeolite $4 \mathrm{~A}$ - Lltem MMMs can be attributed to experimental errors and/or existence of two non-ideal cases in the MMMs depending on the filler loading. In other words, all non-ideal cases may occurred in MMMs simultaneously and in a certain specific filler loading, chain rigidification is dominant, while in other loadings, other non-ideal cases become dominant. Obviously, in this case, deviation of ideal Maxwell model predictions from the experimental data is very significant.

\subsubsection{Prediction of Matrimid5218-CMS MMMs Performance for $\mathrm{CO}_{2} / \mathrm{CH}_{4}$ Separation}

In another case study, MMMs were prepared by incorporation of carbon molecular sieves, CMSs, in Matrimid 5218. The prepared MMMs showed good separation performance for $\mathrm{CO}_{2} / \mathrm{CH}_{4}$ gas mixtures [19]. Regarding to Figure 4], it can be concluded that polymer chains rigidification is more probable for the MMMs performance with increasing filler loading for separation. The results of the modified Maxwell model predictions
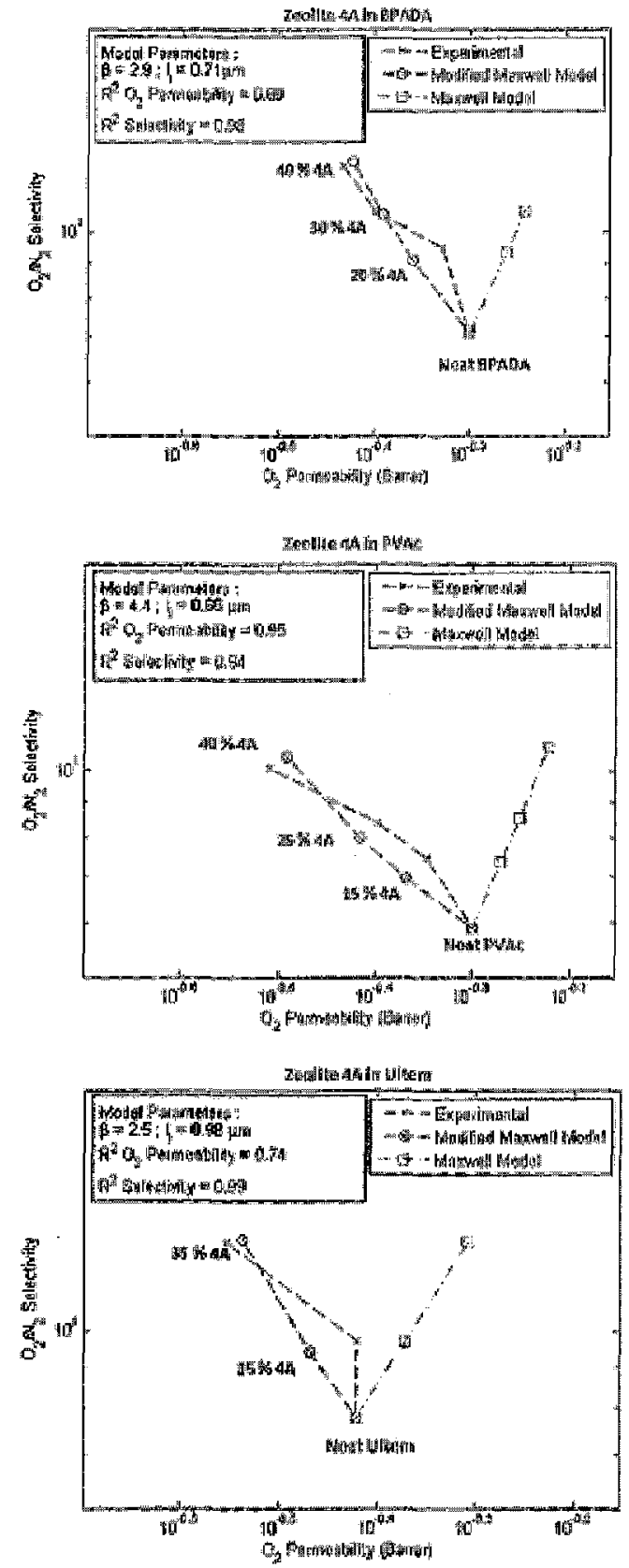

Figure 7 Predictions of the modified Maxwell model of rigidified polymer chains for MMMs formed by incorporation of different zeolite $4 \mathrm{~A}$ loadings in different polymer matrices of PVAc, BAPB-BPADA and Uttem for $\mathrm{O}_{2} / \mathrm{N}_{2}$ separation at $35^{\circ} \mathrm{C}[2]$ 


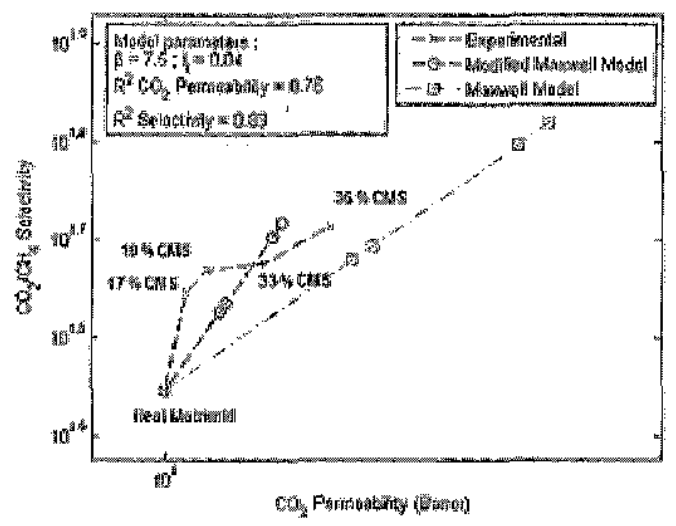

Figure 8 Predictions of the modified Maxwell model of rigidified polymer chains for MMMs formed by incorporation of different CMS loadings in Matrimid 5218 for $\mathrm{CO}_{2} / \mathrm{CH}_{4}$ separation at 35 ${ }^{\circ} \mathrm{C}[15]$

\subsection{MMMs with Void Interface}

\subsubsection{Prediction of Polycarbonate-Zeolite $4 A$ MMMs Performance for $\mathrm{O}_{2 /} / \mathrm{N}_{2}$ Separation}

One of the most key features in MMMs preparation procedure is right selection of filler and polymer to approach MMM separation performance to pass Robeson upper bond limit. As usual, fillers with higher separation performances are incorporated in polymer matrices with lower separation performances with same separation mechanism to enhance the resulted MMMs performance. However, Deger Sen et al. [16] has incorporated less permeable zeolite $4 \mathrm{~A}\left(\mathrm{P}_{\mathrm{O} 2}=0.77\right.$ Barrer [13]) in higher one of polycarbonate $\left(\mathrm{PC}, \mathrm{P}_{\mathrm{O} 2}=1.8\right.$ Barrer) matrix. The prepared MMMs showed both higher separation factors and permeabilities than those expected by ideal Maxwell model. On the other hand, their data cannot be predicted by non of rigidified cases of void case and rigidified chains around the fillers. More investigation indicated that their prepared MMMs have ideal and/or rigidified sections in the lower and upper faces while large voids (as large as the incorporated fillers) were formed within the MMMs bodies (Figure 9) [20]. The integrated upper and lower surfaces of MMM could be attributed to annealing of MMMs at temperatures around the polycarbonate glass transition temperature of $\sim$ $145^{\circ} \mathrm{C}[8]$. Considering a penetrant adsorbed on the upper face of MMM, it diffuses through at least an imagined ideal path (white thick arrow in Figure 9) to pass the MMM. As it can be seem in Figure 9, different medias of ideal and / or rigidified chains, voids, segregated filler particles and finally another ideal and / or rigidified chains maybe experienced by other penetrants. It is the complicated case of chain rigidification, void formation around the fillers, and / or ideal morphologies where cannot be predicted by the current single based models. Employing modified Maxwell model (rigidified chains and formed voids) for this case did not give a meaningful parameter(s).

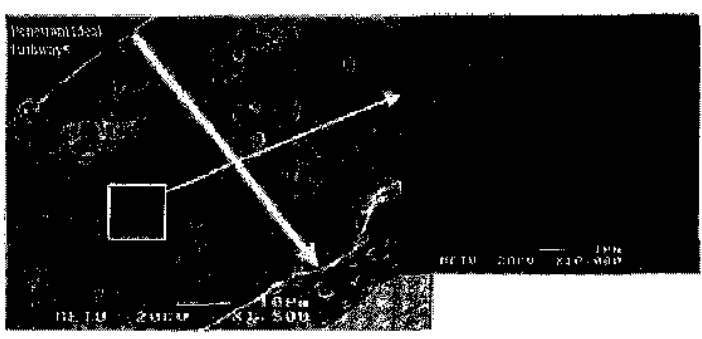

Figure 9 Cross sectional SEM image of PC/ zeolite 4A (20\% loading) [20]

\subsubsection{Prediction of Ultem, Zeolite 4A MMMs Performance for $\mathrm{O}_{2} / \mathrm{N}_{2}$ Separation}

In this case, the results from Figure 4 were used to estimate accuracy of the model predictions. The results of experimental values model predictions showed very good agreement between model predictions and the quantity of the permeability and selectivity. The results of the model for both cases are depicted in Figure 10.

\subsection{MMM's with Chain Rigidification and Partial Pore Blockage}

5.3.1 Prediction of PES-zeolite $4 \mathrm{AMMMS}$ Performance for $\mathrm{O}_{2} / \mathrm{N}_{2}$ Separation Prediction

Yi Li et al, prepared some MMMs using different 

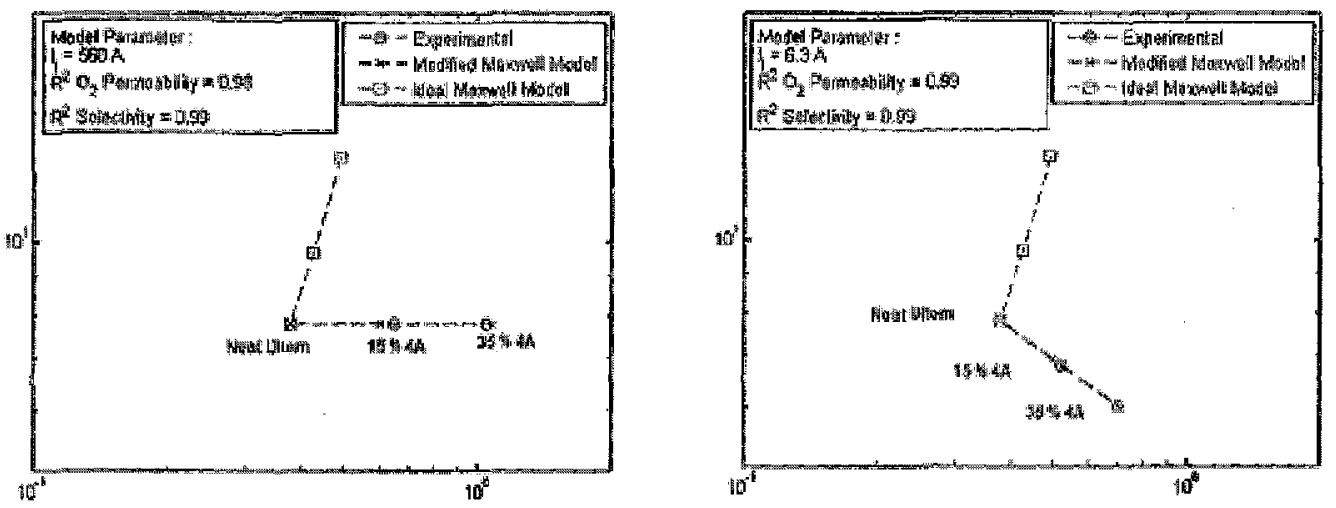

Figure IU I'redictions of the moditled vaxwell model of void interface (Cases II \& III) for MMM formed by incorporation of different zenlite $4 \mathrm{~A}$ lnadinos in Itlem for O $/ \mathrm{N}$ 2 senaratinn at $35^{\circ} \mathrm{C}[1]$

fillex loadings of zeolite 4A in PES as polymer matrix for $\mathrm{O}_{2} / \mathrm{N}_{2}$ separation [21]. Their results showed higher selectivity, but lower permeability indicating non-ideal morphology of chain rigidification around the filler particles. As observed in Figure 11, ideal Maxwell model prediction is so far form experimental data confirming non-ideal morphology in the interface of filler particles. By running the modified Maxwell model for chain rigidification program, it was found that chain rigidification factor $\left(\beta^{\dagger}\right)$ and interface layer thickness $\left(l_{i}^{\prime}\right)$ are 52.6 and 0.19 $\mu m$ respectively, The high value of chain rigidification factor confirms filler particle pore blockage and polymer chain rigidification around the filler particles. Using experimental data in the modified Maxwell model and comparison the results in Figure 11 with those in Figure 4, confirm chain rigidification and partial pore blockage. As observed, the modified Maxwell model can predict filler pore blockage case as well as other nonideal cases.

\subsection{Ideal MMMs}

\subsubsection{Prediction of Ultem-zeolite 4A MMMs Performance for $\mathrm{CO}_{2} / \mathrm{CH}_{4}$ Separation}

In this case, the Modified Maxwell model was employed to predict the Ultem-zeolite 4A MMM separation performance. The results showed that

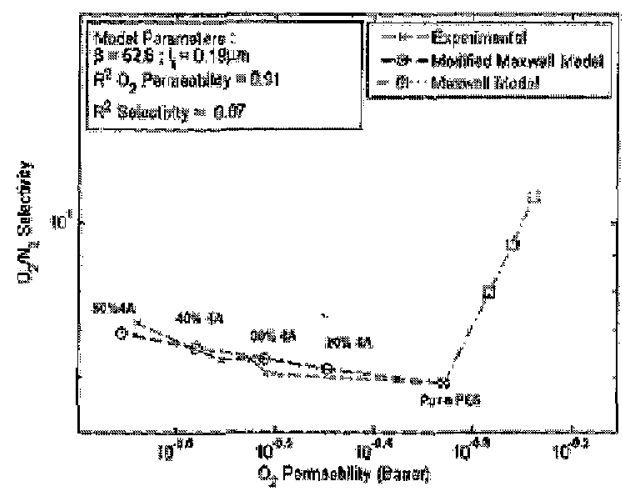

Figure 11 Prediction of Maxwell model for MMMs formed by incorporation of different Zeolite $4 \mathrm{~A}$ loadings in Polyethersulfone $\mathrm{O}_{2} / \mathrm{N}_{2}$ separation at $2.5^{\circ} \mathrm{C}\lceil 211$

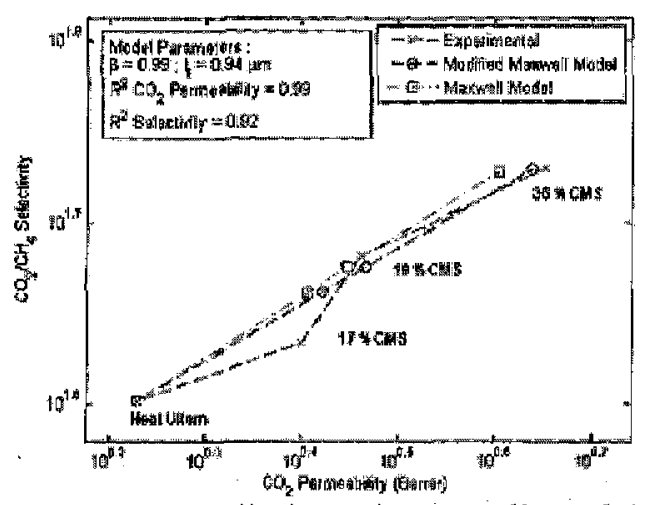

Figure 12 Prediction of Maxwell model for MMMs formed by incorporation of different CMS loadings in Ultem $\mathrm{CO}_{2} / \mathrm{CH}_{4}$ separation at $25^{\circ} \mathrm{C}[15]$ 


\subsection{APPLICATION OF THE MODIFIED BRUGGEMAN MODEL FOR MMMS PREDICTION SEPARATION PERFORMANCE}

\subsection{MMMs with Rigidified Interface}

\subsubsection{Prediction of MMMs with Zeolite $4 A$ Fillers Performance for $\mathrm{O}_{2} / \mathrm{N}_{2}$ Separation}

In this case, the results of several MMM's were used by the modified Bruggeman model and the results are presented in Figure 13. As observed the results are well in agreement by the experimental data and the results shown good prediction performance for this case.

\subsubsection{Prediction of Matrimid5218-CMS} MMMs Performance for $\mathrm{CO}_{2} / \mathrm{CH}_{4}$ Separation

In this case study, the experimental results obtained by [15] were used to estimate accuracy of the modified Bruggeman model for this type of the MMMs and the results are shown in Figure 14. As observed, the results show an improvement in accuracy of the model predictions.
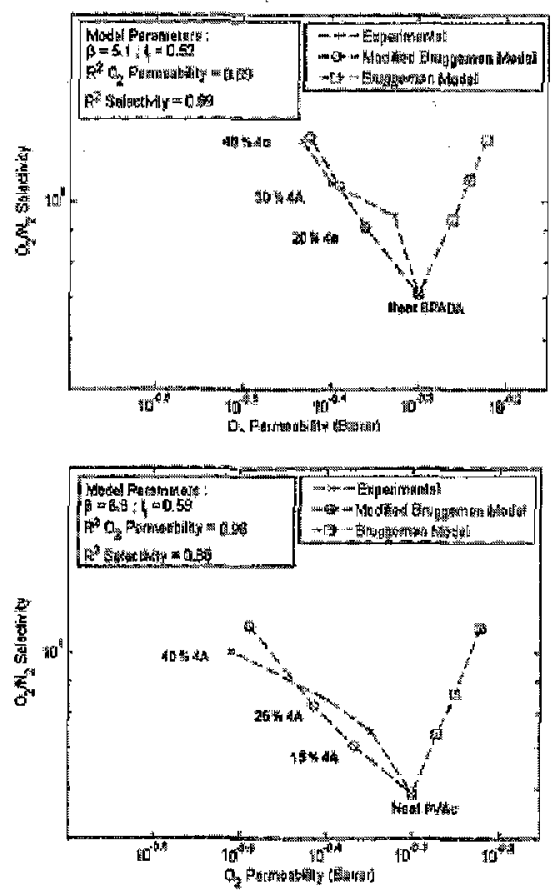

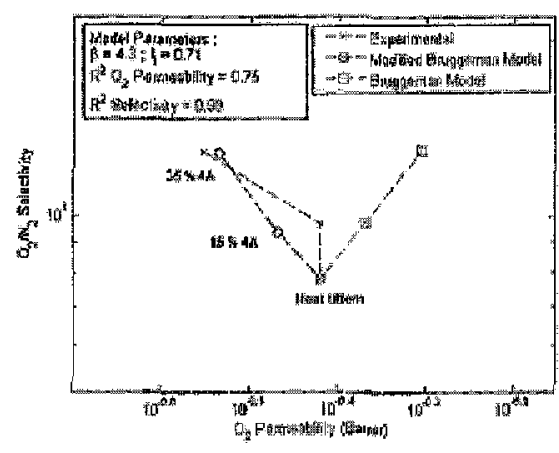

Figure 13 Predictions of the modified Bruggeman model of rigidified polymer chains for MMMs formed by incorporation of different zeolite $4 \mathrm{~A}$ loadings in different polymer matrices of PVAc, BAPB-BPADA and Ultem for $\mathrm{O} 2 / \mathrm{N} 2$ separation at $35^{\circ} \mathrm{C}[2]$

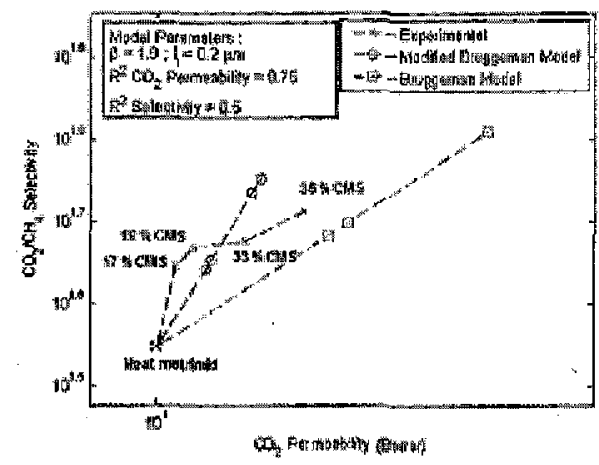

Figure 14 Predictions of the modified Bruggeman model of rigidified polymer chains for MMMs formed by incorporation of different CMS loadings in Matrimid 5218 for $\mathrm{CO}_{2} /$ $\mathrm{CH}_{4}$ separation at $35^{\circ} \mathrm{C}[15]$

\subsection{MMMs with Void Interface}

\subsubsection{Prediction of Polycarbonate-Zeolite $4 A$ MMMs Performance for $\mathrm{O}_{2} / \mathrm{N}_{2}$ Separation}

Similar with the modified Maxwell model, the modified Bruggeman model also cannot predict the experimental data obtained by Sen et al. This is due to the simultaneous occurring of non-ideal phenomena. 

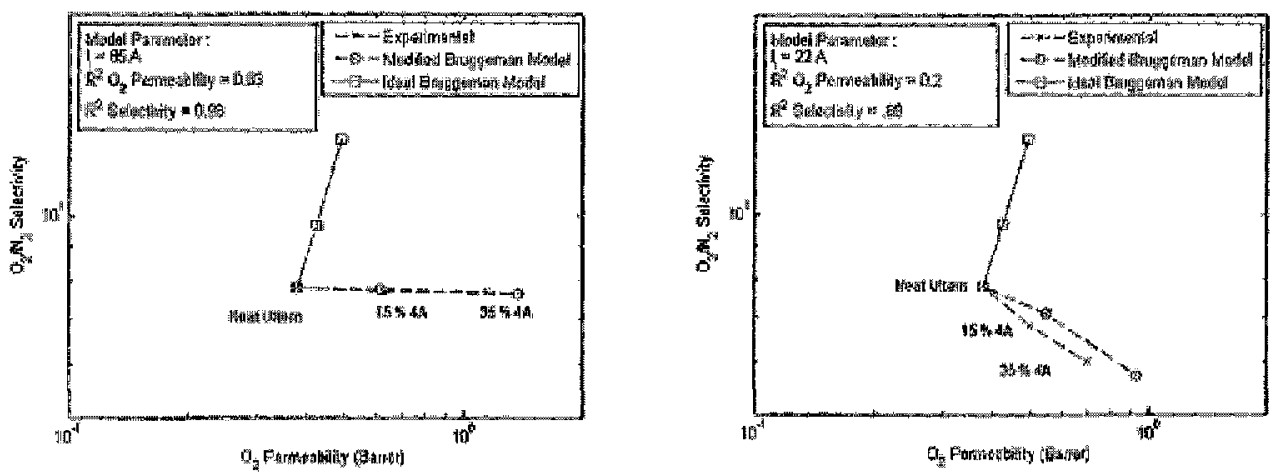

Figure 15 Predictions of the modified Bruggeman model of void interface(Cases II \& III) for MMM formed by incorporation of different zeolite $4 \mathrm{~A}$ loadings in Ultem for $\mathrm{O} 2 / \mathrm{N} 2$ separation at $35^{\circ} \mathrm{C}[[1]$

\subsubsection{Prediction of Ultem, Zeolite 4A MMMs Performance for $\mathrm{O}_{2} / \mathrm{N}_{2}$ Separation}

The results from Figure 4 were used and the model predictions were depicted in Figure 15. As observed the results of the model in both cases are in good agreement with the data extracted from Figure 4.

\subsection{MMM's with Chain Rigidification and Partial Pore Blockage}

\subsubsection{Prediction of PES-zeolite 4A. MMMs} Performance for $\mathrm{O}_{2} / \mathrm{N}_{2}$ Separation Prediction

In this case, the experimental results obtained by Li et al. [21] were applied and modeled using the modified Bruggeman model and the results are reported in Figure 16. The results show very good agreement between experimental data and the model predictions.

\subsection{Ideal MMMs}

\subsubsection{Prediction of Ultem-zeolite $4 A$ MMMs Performance for $\mathrm{CO}_{2} / \mathrm{CH}_{4}$ Separation}

The results of the CMS-Ultem MMMs performance obtained by Vu et al. [15] were used for this case and the results of the model predictions are shown in Figure 17.

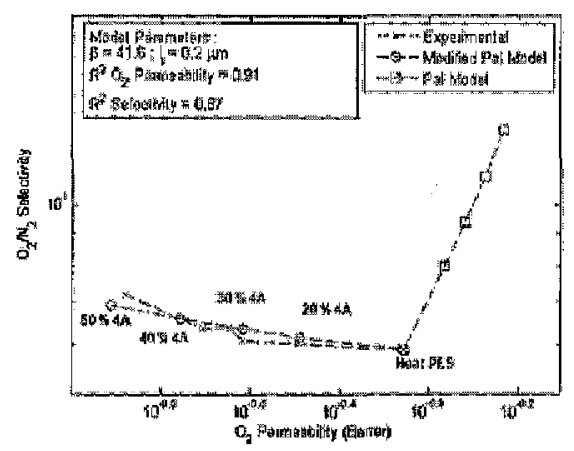

Figure 16 Prediction of modified Bruggeman model for MMMs formed by incorporation of different Zeolite $4 \mathrm{~A}$ loadings in Polyethersulfone $\mathrm{O}_{2} / \mathrm{N}_{2}$ separation at $25^{\circ} \mathrm{C}[21]$

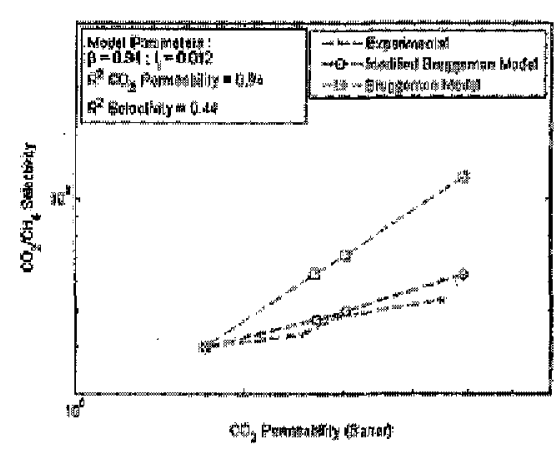

Figure 17 Prediction of modified Bruggeman model for MMMs formed by incorporation of different CMS loadings in Ultem $\mathrm{CO}_{2} / \mathrm{CH}_{4}$ separation at $25^{\circ} \mathrm{C}[15]$ 
Table 2 AARE \% values of the modified models in the current studies

\begin{tabular}{llllllll}
\hline $\begin{array}{l}\text { Case } \\
\text { Studies }\end{array}$ & $\begin{array}{l}\text { Ideal } \\
\text { CMS- } \\
\text { Ultem }\end{array}$ & $\begin{array}{l}\text { BPADA- } \\
\text { 4A }\end{array}$ & $\begin{array}{l}\text { Rigidi- } \\
\text { fication } \\
\text { PVAc-4A }\end{array}$ & Ultem-4A & $\begin{array}{l}\text { Matrimid- } \\
\text { CMS }\end{array}$ & $\begin{array}{l}\text { Partially pore Void interface } \\
\text { blockage Pes- Ultem-4A } \\
\text { 4A }\end{array}$ \\
\hline $\begin{array}{l}\text { Modified } \\
\text { Maxwell }\end{array}$ & 2.39 & 2.78 & 3.23 & 4.77 & 3.09 & 5.67 & 3.40 \\
$\begin{array}{l}\text { Modified } \\
\text { Bruggeman }\end{array}$ & 5.18 & 2.71 & 3.27 & 4.83 & 3.09 & 5.77 & 7.18 \\
\hline
\end{tabular}

\subsection{COMPARISON OF THE MODIFIED MODELS PREDICTION ACCURACY}

The results showed that applying the modified models leads to better predictions compared with the ideal models and these improvements in the predictions ability are the result of using the $3^{\text {rd }}$ phase properties called the interface phase.

The applied models in the current study were Maxwell and Bruggeman models. Modification of these models results always in high prediction accuracy.

The results of the AARE \% values for different cases are shown in Table 2.

As can be seen in Table 2 the prediction accuracy of the both modified models are relatively high and in more cases, the modified Maxwell model represents better predictions than the modified Bruggeman model, and as mentioned before this is due to the fact that the Bruggeman model is an implicit model and there are several limitations for converging the results using this model. As a result, the modified Maxwell model seems better for prediction of the MMMs performance.

\subsection{CONCLUSION}

MMMs as new membrane generations have made many hopes to overcome both polymeric and molecular sieve membranes shortcomings by incorporating fine molecular sieves to polymeric matrices to approach desired economical separation performances over the Robeson upper bound limit. Different ideal models, e. g. Maxwell and Bruggeman models, have been developed to predict MMMs separation performances knowing those of neat polymeric membranes and incorporated fillers and filler loading. The ideal models cannot predict all MMMs performance due to non-ideal morphologies might occurred in MMMs structure such as voids formation and chain rigidification around the fillers and partial or total fillers pores blockage. These defects should be considered in predictive models to provide better predictions of MMMs. Some Modified models were developed to consider these nonideal morphologies as the third phase of interface as voids and rigidified polymer region.

The modified models consider only one nonideal defect at a time, however, there may be two or more non-ideal morphologies encountered simultaneously and one of them becomes controlling at a certain filler loading and the others become predominant at different filler loadings. Meanwhile, the modified models were employed to fit the experimental data using the least square method. The findings showed that the models can predict experimental data in some cases.

\section{REFERENCES}

[1] Theodore T. Moore and William J. Koros. 2005. Non-ideal Effects in Organicinorganic Materials for Gas Separation Membranes. Journal of Molecular Structure. 739(1-3): 87-98. 
[2] Tai-Shung Chung, et al. 2007. Mixed Matrix Membranes (MMMs) Comprising Organic Polymers with Dispersed Inorganic Fillers for Gas Separation. Progress in Polymer Science. 32(4):483-507.

[3] Theodore T. Moore, et al. 2004. Hybrid Membrane Materials Comprising Organic Polymers with Rigid Dispersed Phases. AIChE Journal. $50(2): 311-321$.

[4] Lloyd M. Robeson. 1991. Correlation of Separation Factor Versus Permeability for Polymeric Membranes. Journal of Membrane Science. 62(2): 165-185.

[5] Biruh Shimekit, Hilmi Mukhtar, and Thanapalan Murugesan. 2011. Prediction of the Relative Permeability of Gases in Mixed Matrix Membranes. Journal of Membrane Science. 373(1-2): 152-159.

[6] Rajinder Pal. 2008. Permeation Models for Mixed Matrix Membranes. Journal of Colloid and Interface Science. 317(1): 191-198.

[7] Rajiv Mahajan, et al. 2002. Challenges in Forming Successful Mixed Matrix Membranes with Rigid Polymeric Materials. Journal of Applied Polymer Science. 86(4): 881-890.

[8] Lan Ying Jiang, Tai-Shung Chung, and Raj Rajagopalan. 2007. Matrimid®/MgO mixed Matrix Membranes for Pervaporation. AIChE Journal. 53(7): 1745-1757.

[9] S. Birgül Tantekin-Ersolmaz, et al. 2000. Effect of Zeolite Particle Size on the Performance of Polymer-zeolite Mixed Matrix Membranes. Journal of Membrane Science. 175(2): 285-288.

[10] V. Bhardwaj. 2003. Polysulfone Hollow Fiber Gas Separation Membranes Filled with Submicron Particles. New York Academy of Sciences. 984: 318-328.

[11] Murat G. Süer, Nurcan Baç, and Levent Yilmaz. 1994. Gas Permeation Characteristics of Polymer-zeolite Mixed Matrix Membranes. Journal of Membrane Science. 91(1-2): 77-86.

[12] S. A. Hashemifard, A. F. Ismail, and T.
Matsuura. 2010. A New Theoretical Gas Permeability Model using Resistance Modeling for Mixed Matrix Membrane Systems. Journal of Membrane Science. 350(1-2): 259-268.

[13] R. Mahajan T.moore, D.vu,W.koros. 2004. Hybrid Membrane Materials Comprising Organic Polymers With Rigid DispersedPhases. AIChe. 50: 311.

[14] W. Yang. 2005. Applied Numerical Methods using MATLAB. Wiley-Interscience.

[15] De Q. Vu, William J. Koros, and Stephen J. Miller. 2003. Mixed Matrix Membranes using Carbon Molecular Sieves: II. Modeling Permeation Behavior. Joumal of Membrane Science. 211(2): 335-348.

[16] Deger Sen, Halil Kalipcilar, and Levent Yilmaz. 2006. Development of Zeolite Filled Polycarbonate Mixed Matrix Gas Separation Membranes. Desalination. 200(1-3): 222-224.

[17] W.J.Koros R. Mahajan. 2002. Mixed Matrix Membrane Materials with Glassy Polymers.Part 1. Polymer Engineering and Science. 42.

[18] W. J.Koros R. Mahajan. 2000. Factors Controlling Successfull Formation of Mixed-Matrix Gas Separation Materials. Ind.Eng.Chem. 39.

[19] De Q. Vu, William J. Koros, and Stephen J. Miller. 2003. Mixed Matrix Membranes using Carbon Molecular Sieves: I. Preparation and Experimental Results. Journal of Membrane Science. 211(2): 311334.

[20] Deger Sen, Halil KalIpçIlar, and Levent Yilmaz. 2007. Development of Polycarbonate based Zeolite 4A Filled Mixed Matrix Gas Separation Membranes. Journal of Membrane Science. 303 (1-2): 194-203.

[21] Yi Li, et al. 2005. The Effects of Polymer Chain Rigidification, Zeolite Pore Size and Pore Blockage on Polyethersulfone (PES)Zeolite A Mixed Matrix Membranes. Journal of Membrane Science. 260(1-2): 45-55. 